TITLE: HEXAHEDRON, WEDGE, TETRAHEDRON, AND PYRAMID DIFFUSION OPERATOR DISCRETIZATION

AUTHOR(S): $\quad$ Randy M. Roberts

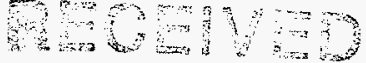

bing 2109

OSTI

SUBMITTED TO: $\quad$ For electronic distribution upon request.

DSTRIIUTION OF THS DOCUMENT is UNLMIED
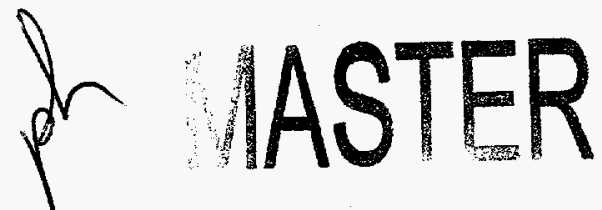

By acceptance of this article, the publisher recognizes that the U.S. Government retains a nonexclusive royalty-free license to publish or reproduce the published form of this contribution or to allow others to do so, for U.S. Government purposes.

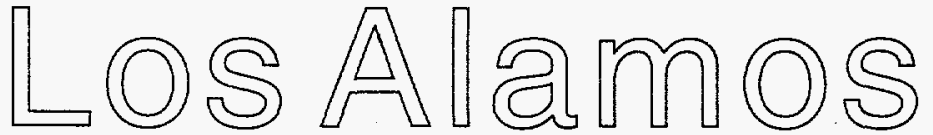




\section{DISCLAMMER}

Portions of this document may be illegible in electronic image products. Images are produced from the best available original document. 


\section{DISCLAIMER}

This report was prepared as an account of work sponsored by an agency of the United States Government. Neither the United States Government nor any agency thereof, nor any of their employees, makes any warranty, express or implied, or assumes any legal liability or responsibility for the accuracy, completeness, or usefulness of any information, apparatus, product, or process disclosed, or represents that its use would not infringe privately owned rights. Reference herein to any specific commercial product, process, or service by trade name, trademark, manufacturer, or otherwise does not necessarily constitute or imply its endorsement, recommendation, or favoring by the United States Government or any agency thereof. The views and opinions of authors expressed herein do not necessarily state or reflect those of the United States Government or any agency thereof. 


\title{
Hexahedron, Wedge, Tetrahedron, and Pyramid Diffusion Operator Discretization
}

\author{
Randy M. Roberts
}

August 6, 1996 


\section{General Variational Function}

We are looking for the solution of the diffusion equation, $\phi(\vec{x})$, by finding the extrema of the following functional

$$
\Gamma[\phi]=\int\left(\frac{1}{2} D \vec{\nabla} \phi \cdot \vec{\nabla} \phi+\frac{1}{2} \sigma_{a} \phi^{2}-Q \phi\right) d^{3} x .
$$

At this point I will concentrate only on the first term in the integral, and so take

$$
\Gamma[\phi]=\int \frac{1}{2} D \vec{\nabla} \phi \cdot \vec{\nabla} \phi d^{3} x .
$$

Also surface integrals arising from the boundaries of the problem will be ignored. These will be treated in a later section.

We will now discretize the above equation in terms of the values for $\phi(\vec{x})$ at each of the corners of the cell, $\phi_{i}$. The functional, $\Gamma$, is replaced by a function of these corner values

$$
\Gamma\left(\phi_{1}, \phi_{2}, \ldots\right)=\frac{1}{2} D \sum_{k} w_{k}\left\|\vec{\nabla}_{k} \phi\right\|^{2}
$$

where the sum is over the corners of the cell, and $\vec{\nabla}_{k} \phi$ is itself a function of the $\phi_{i} \mathrm{~s}$, and $w_{k}$ are weights that sum to the volume of the cell. The form of the expression for $\vec{\nabla}_{i} \phi$ is

$$
\vec{\nabla}_{i} \phi=\sum_{k^{\prime}}\left(\phi_{k^{\prime}}-\phi_{i}\right) \vec{\alpha}_{i k^{\prime}}
$$

The sum extends only over the 3 nearest neighbors of the point, $i$. The $\vec{\alpha}_{i k^{\prime}}$ is defined by

$$
\vec{\alpha}_{i j}=\frac{\vec{r}_{i k} \times \vec{r}_{i l}}{\vec{r}_{i j} \cdot\left(\vec{r}_{i k} \times \vec{r}_{i l}\right)},
$$

where $k$ and $l$ are the two neighbor corners of $i$ that are not $j$.

The extrema of $\Gamma$ is found by solving the following set of simultaneous equations for the $\phi_{i} s$

$$
\frac{\partial \Gamma}{\partial \phi_{i}}=0
$$

Since $\Gamma$ has the form of Equation 3 with $\vec{\nabla}_{i} \phi$ in the form of Equation 4 , the above equation becomes

$$
\sum_{j} \frac{\partial^{2} \Gamma}{\partial \phi_{i} \partial \phi_{j}} \phi_{j}=0
$$

Substituting the above expressions for $\Gamma$ and $\vec{\nabla}_{i} \phi$ we arrive at an expression for the matrix element, $A_{i j}$

$$
A_{i j}=\frac{\partial^{2} \Gamma}{\partial \phi_{i} \partial \phi_{j}}=D \sum_{k} w_{k} \frac{\partial \vec{\nabla}_{k} \phi}{\partial \phi_{i}} \cdot \frac{\partial \vec{\nabla}_{k} \phi}{\partial \phi_{j}} .
$$

In the following sections we will investigate this matrix for the hexahedron, wedge, tetrahedron, and pyramid cells. 


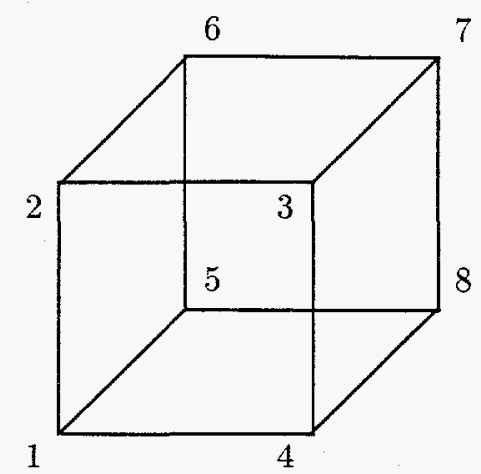

Figure 1: The corner numbering scheme for the hexahedron.

\section{Hexahedron Matrix Elements}

For the hexahedron we will be looking at the following matrix elements, $A_{11}$, $A_{12}, A_{16}$, and $A_{17}$. By looking at these matrix elements we have covered those matrix elements that are fundamentally different. All other matrix elements may be found by a suitable topology-preserving permutation of the corner indices.

For the hexahedron the expressions for the $\vec{\nabla}_{i} \phi$ s are

$$
\begin{aligned}
\vec{\nabla}_{1} \phi & =\left(\phi_{2}-\phi_{1}\right) \vec{\alpha}_{12}+\left(\phi_{4}-\phi_{1}\right) \vec{\alpha}_{14}+\left(\phi_{5}-\phi_{1}\right) \vec{\alpha}_{15} \\
\vec{\nabla}_{2} \phi & =\left(\phi_{1}-\phi_{2}\right) \vec{\alpha}_{21}+\left(\phi_{3}-\phi_{2}\right) \vec{\alpha}_{23}+\left(\phi_{6}-\phi_{2}\right) \vec{\alpha}_{26} \\
\vec{\nabla}_{3} \phi & =\left(\phi_{2}-\phi_{3}\right) \vec{\alpha}_{32}+\left(\phi_{4}-\phi_{3}\right) \vec{\alpha}_{34}+\left(\phi_{7}-\phi_{3}\right) \vec{\alpha}_{37} \\
\vec{\nabla}_{4} \phi & =\left(\phi_{1}-\phi_{4}\right) \vec{\alpha}_{41}+\left(\phi_{3}-\phi_{4}\right) \vec{\alpha}_{43}+\left(\phi_{8}-\phi_{4}\right) \vec{\alpha}_{48} \\
\vec{\nabla}_{5} \phi & =\left(\phi_{1}-\phi_{5}\right) \vec{\alpha}_{51}+\left(\phi_{6}-\phi_{5}\right) \vec{\alpha}_{56}+\left(\phi_{8}-\phi_{5}\right) \vec{\alpha}_{58} \\
\vec{\nabla}_{6} \phi & =\left(\phi_{2}-\phi_{6}\right) \vec{\alpha}_{62}+\left(\phi_{5}-\phi_{6}\right) \vec{\alpha}_{65}+\left(\phi_{7}-\phi_{6}\right) \vec{\alpha}_{67} \\
\vec{\nabla}_{7} \phi & =\left(\phi_{3}-\phi_{7}\right) \vec{\alpha}_{73}+\left(\phi_{6}-\phi_{7}\right) \vec{\alpha}_{76}+\left(\phi_{8}-\phi_{7}\right) \vec{\alpha}_{78} \\
\vec{\nabla}_{8} \phi & =\left(\phi_{4}-\phi_{8}\right) \vec{\alpha}_{84}+\left(\phi_{5}-\phi_{8}\right) \vec{\alpha}_{85}+\left(\phi_{7}-\phi_{8}\right) \vec{\alpha}_{87}
\end{aligned}
$$

Only $\vec{\nabla}_{k} \phi$ for $k \in\{1,2,4,5\}$ will contribute to the calculation of $A_{1 j}$, therefore,

$$
\begin{aligned}
A_{11}= & D\left[w_{1}\left\|\frac{\partial \vec{\nabla}_{1} \phi}{\partial \phi_{1}}\right\|^{2}+w_{2}\left\|\frac{\partial \vec{\nabla}_{2} \phi}{\partial \phi_{1}}\right\|^{2}+w_{4}\left\|\frac{\partial \vec{\nabla}_{4} \phi}{\partial \phi_{1}}\right\|^{2}\right. \\
& \left.+w_{5}\left\|\frac{\partial \vec{\nabla}_{5} \phi}{\partial \phi_{1}}\right\|^{2}\right] \\
= & D\left[w_{1}\left\|\vec{\alpha}_{12}+\vec{\alpha}_{14}+\vec{\alpha}_{15}\right\|^{2}+w_{2}\left\|\vec{\alpha}_{21}\right\|^{2}+w_{4}\left\|\vec{\alpha}_{41}\right\|^{2}\right.
\end{aligned}
$$




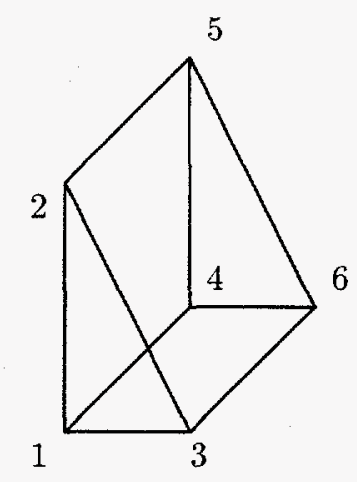

Figure 2: The corner numbering scheme for the wedge.

$$
\begin{aligned}
& \left.+w_{5}\left\|\vec{\alpha}_{51}\right\|^{2}\right] \\
A_{12}= & D\left[w_{1} \frac{\partial \vec{\nabla}_{1} \phi}{\partial \phi_{1}} \cdot \frac{\partial \vec{\nabla}_{1} \phi}{\partial \phi_{2}}+w_{2} \frac{\partial \vec{\nabla}_{2} \phi}{\partial \phi_{1}} \cdot \frac{\partial \vec{\nabla}_{2} \phi}{\partial \phi_{2}}\right] \\
= & -D\left[w_{1}\left(\vec{\alpha}_{12}+\vec{\alpha}_{14}+\vec{\alpha}_{15}\right) \cdot \vec{\alpha}_{12}+w_{2} \vec{\alpha}_{21} \cdot\left(\vec{\alpha}_{21}+\vec{\alpha}_{23}+\vec{\alpha}_{26}\right)\right] \\
A_{16}= & D\left[w_{2} \frac{\partial \vec{\nabla}_{2} \phi}{\partial \phi_{1}} \cdot \frac{\partial \vec{\nabla}_{2} \phi}{\partial \phi_{6}}+w_{5} \frac{\partial \vec{\nabla}_{5} \phi}{\partial \phi_{1}} \cdot \frac{\partial \vec{\nabla}_{5} \phi}{\partial \phi_{6}}\right] \\
= & D\left[w_{2} \vec{\alpha}_{21} \cdot \vec{\alpha}_{26}+w_{5} \vec{\alpha}_{51} \cdot \vec{\alpha}_{56}\right] \\
A_{17}= & 0 .
\end{aligned}
$$

In general this leads to a 19 point diffusion operator. For a rectangular hexahedral mesh it is easy to see that $A_{16}$ vanishes, leaving one with the standard 7 point operator.

\section{$3 \quad$ Wedge Matrix Elements}

For the wedge we will be looking at the following matrix elements, $A_{11}, A_{12}$, $A_{14}$, and $A_{15}$. By looking at these matrix elements we have covered those matrix elements that are fundamentally different. All other matrix elements may be found by a suitable topology-preserving permutation of the corner indices.

For the wedge the expressions for the $\vec{\nabla}_{i} \phi$ s are

$$
\begin{aligned}
& \vec{\nabla}_{1} \phi=\left(\phi_{2}-\phi_{1}\right) \vec{\alpha}_{12}+\left(\phi_{3}-\phi_{1}\right) \vec{\alpha}_{13}+\left(\phi_{4}-\phi_{1}\right) \vec{\alpha}_{14} \\
& \vec{\nabla}_{2} \phi=\left(\phi_{1}-\phi_{2}\right) \vec{\alpha}_{21}+\left(\phi_{3}-\phi_{2}\right) \vec{\alpha}_{23}+\left(\phi_{5}-\phi_{2}\right) \vec{\alpha}_{25} \\
& \vec{\nabla}_{3} \phi=\left(\phi_{1}-\phi_{3}\right) \vec{\alpha}_{31}+\left(\phi_{2}-\phi_{3}\right) \vec{\alpha}_{32}+\left(\phi_{6}-\phi_{3}\right) \vec{\alpha}_{36} \\
& \vec{\nabla}_{4} \phi=\left(\phi_{1}-\phi_{4}\right) \vec{\alpha}_{41}+\left(\phi_{5}-\phi_{4}\right) \vec{\alpha}_{45}+\left(\phi_{6}-\phi_{4}\right) \vec{\alpha}_{46}
\end{aligned}
$$




$$
\begin{aligned}
& \vec{\nabla}_{5} \phi=\left(\phi_{2}-\phi_{5}\right) \vec{\alpha}_{52}+\left(\phi_{4}-\phi_{5}\right) \vec{\alpha}_{54}+\left(\phi_{6}-\phi_{5}\right) \vec{\alpha}_{56} \\
& \vec{\nabla}_{6} \phi=\left(\phi_{3}-\phi_{6}\right) \vec{\alpha}_{63}+\left(\phi_{4}-\phi_{6}\right) \vec{\alpha}_{64}+\left(\phi_{5}-\phi_{6}\right) \vec{\alpha}_{65}
\end{aligned}
$$

Following the same procedures as for the hexahedron, we find the matrix elements to be

$$
\begin{aligned}
A_{11}= & D\left[w_{1}\left\|\vec{\alpha}_{12}+\vec{\alpha}_{13}+\vec{\alpha}_{14}\right\|^{2}+w_{2}\left\|\vec{\alpha}_{21}\right\|^{2}+w_{3}\left\|\vec{\alpha}_{31}\right\|^{2}\right. \\
& \left.+w_{4}\left\|\vec{\alpha}_{41}\right\|^{2}\right] \\
A_{12}= & -D\left[w_{1}\left(\vec{\alpha}_{12}+\vec{\alpha}_{13}+\vec{\alpha}_{14}\right) \cdot \vec{\alpha}_{12}+w_{2} \vec{\alpha}_{21} \cdot\left(\vec{\alpha}_{21}+\vec{\alpha}_{23}+\vec{\alpha}_{25}\right)\right] \\
A_{14}= & -D\left[w_{1}\left(\vec{\alpha}_{12}+\vec{\alpha}_{13}+\vec{\alpha}_{14}\right) \cdot \vec{\alpha}_{14}+w_{4} \vec{\alpha}_{41} \cdot\left(\vec{\alpha}_{41}+\vec{\alpha}_{45}+\vec{\alpha}_{46}\right)\right] \\
A_{15}= & D\left[w_{2} \vec{\alpha}_{21} \cdot \vec{\alpha}_{25}+w_{4} \vec{\alpha}_{41} \cdot \vec{\alpha}_{45}\right] .
\end{aligned}
$$

One can see that all the corner points couple to all of the others. In a general logically orthogonal grid connected as wedges this would yield a 15 point diffusion operator. If the wedge was created from an orthogonal grid then it is easy to see that $A_{15}$ vanishes, and that each point is coupled only with its neighbors. This again yields the standard 7 point operator.

At one point we were considering treating the wedge as a degenerate hexahedron, e.g. a hexahedron with points 2 and 6 approaching points 1 and 5, respectively. This approach will not yield the same matrix elements that were derived in this section. This can be seen by looking at the expression for $A_{15}$ for the hexahedron, corresponding to $A_{14}$ for the wedge, with Equations 9-16 modified as follows,

$$
\begin{aligned}
& \phi_{2} \rightarrow \phi_{1} \\
& \phi_{6} \rightarrow \phi_{5} \\
& \vec{r}_{2} \rightarrow \vec{r}_{1} \\
& \vec{r}_{6} \rightarrow \vec{r}_{5} \\
& w_{1}=0 \\
& w_{2}=0 \\
& w_{5}=0 \\
& w_{6}=0 .
\end{aligned}
$$

The reason for setting the $w_{k}$ to zero for points $1,2,5$, and 6 is that the $w_{k}$ represent the volume weight of those points on the hexahedron. When $w_{k}$ goes to zero as the parallelpiped volume of the corner then the $w_{k}\left\|\vec{\nabla}_{k} \phi\right\|^{2}$ terms go to zero. By inspection of $\vec{\nabla}_{3} \phi, \vec{\nabla}_{4} \phi, \vec{\nabla}_{7} \phi$, and $\vec{\nabla}_{8} \phi$, modified as above, one sees that there is no coupling between $\phi_{1}$ and $\phi_{5}$; therefore, the $A_{15}$ matrix element is zero. For the wedge $A_{14}$ is not, in general, zero. This shows that the wedge cannot be treated as a degenerate hex to obtain the wedge results of this section. 


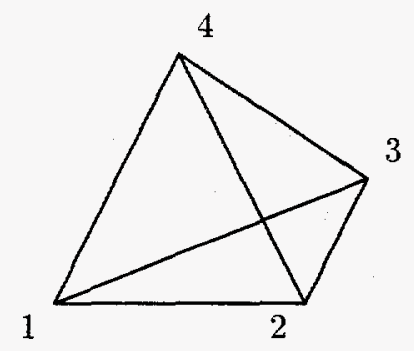

Figure 3: The corner numbering scheme for the tetrahedron.

\section{Tetrahedron Matrix Elements}

For the tetrahedron we will only have to look at the $A_{11}$ and $A_{12}$ matrix elements. They are

$$
\begin{aligned}
A_{11}= & D\left[w_{1}\left\|\vec{\alpha}_{12}+\vec{\alpha}_{13}+\vec{\alpha}_{14}\right\|^{2}+w_{2}\left\|\vec{\alpha}_{21}\right\|^{2}+w_{3}\left\|\vec{\alpha}_{31}\right\|^{2}\right. \\
& \left.+w_{4}\left\|\vec{\alpha}_{41}\right\|^{2}\right] \\
A_{12}= & D\left[-w_{1}\left(\vec{\alpha}_{12}+\vec{\alpha}_{13}+\vec{\alpha}_{14}\right) \cdot \vec{\alpha}_{12}-w_{2} \vec{\alpha}_{21} \cdot\left(\vec{\alpha}_{21}+\vec{\alpha}_{23}+\vec{\alpha}_{24}\right)\right. \\
& \left.+w_{3} \vec{\alpha}_{31} \cdot \vec{\alpha}_{32}+w_{4} \vec{\alpha}_{41} \cdot \vec{\alpha}_{42}\right] .
\end{aligned}
$$

Up until this point the only specification for $w_{i}$ was that its sum over corners equals the volume of the cell. It has been shown that a very good choice of $w_{i}$ for a cell type is

$$
w_{i}=\frac{V_{i}}{\sum_{j} V_{j}} V
$$

where $V$ is the volume of the cell, and $V_{i}$ is the volume of the parallelepiped formed by the three cell edges emanating from the corner $i$. For a tetrahedron $w_{i}$ is independent of the corner,

$$
w_{i}=\frac{1}{4} V
$$

With this choice of $w_{i}$ the matrix element expressions simplify to

$$
\begin{aligned}
& A_{11}=\frac{1}{9} \frac{D}{V}\left\|\vec{A}_{1}\right\|^{2} \\
& A_{12}=\frac{1}{9} \frac{D}{V} \vec{A}_{1} \cdot \vec{A}_{2},
\end{aligned}
$$

where $\vec{A}_{i}$ is the area vector for the triangle face opposite corner $i$. 


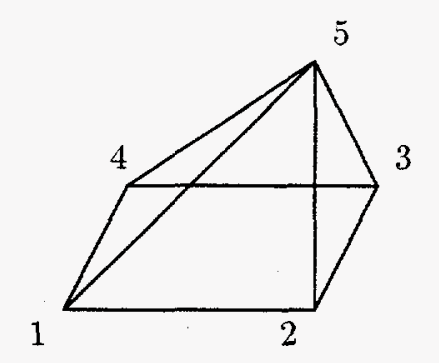

Figure 4: The corner numbering scheme for the pyramid.

In anticipation of the pyramid results we will modify our notation for the tetrahedron matrix elements to be explicit about which points $w_{i}$ and $\vec{A}_{i}$ refer. We will define $V_{i j k l}$ to be the volume of the tetrahedron with corners $i, j, k, l$, and $\vec{A}_{i j k}$ to be the area vector of triangle $i j, k$. The matrix elements for the tetrahedron containing points $1,2,3,4$ become

$$
\begin{aligned}
A_{11}^{1234} & =\frac{1}{9} \frac{D}{V_{1234}}\left\|\vec{A}_{234}\right\|^{2} \\
A_{12}^{1234} & =\frac{1}{9} \frac{D}{V_{1234}} \vec{A}_{234} \cdot \vec{A}_{143} .
\end{aligned}
$$

\section{Pyramid Matrix Elements}

In this section we calculate the matrix elements of the pyramid as the average of the matrix elements from the 4 tetrahedra, $\{1,2,4,5\},\{2,3,4,5\},\{1,3,4$, $5\}$, and $\{1,2,3,5\}$,

$$
A_{i j}=\frac{1}{4}\left[A_{i j}^{1245}+A_{i j}^{2345}+A_{i j}^{1345}+A_{i j}^{1235}\right] .
$$

Due to the linearity of derivatives the same matrix elements would be recovered by first averaging the four tetrahedron functionals, $\Gamma^{i j k l}$, and then performing the variational procedure.

The distinct matrix elements for the pyramid are $A_{11}, A_{12}, A_{13}, A_{15}$, and $A_{55}$,

$$
\begin{aligned}
& A_{11}=\frac{D}{36}\left[\frac{1}{V_{1245}}\left\|\vec{A}_{245}\right\|^{2}+\frac{1}{V_{1345}}\left\|\vec{A}_{345}\right\|^{2}+\frac{1}{V_{1235}}\left\|\vec{A}_{235}\right\|^{2}\right] \\
& A_{12}=\frac{D}{36}\left[\frac{1}{V_{1245}} \vec{A}_{245} \cdot \vec{A}_{154}+\frac{1}{V_{1235}} \vec{A}_{235} \cdot \vec{A}_{153}\right]
\end{aligned}
$$




$$
\begin{aligned}
A_{13}= & \frac{D}{36}\left[\frac{1}{V_{1345}} \vec{A}_{345} \cdot \vec{A}_{154}+\frac{1}{V_{1235}} \vec{A}_{235} \cdot \vec{A}_{125}\right] \\
A_{15}= & \frac{D}{36}\left[\frac{1}{V_{1245}} \vec{A}_{245} \cdot \vec{A}_{142}+\frac{1}{V_{1345}} \vec{A}_{345} \cdot \vec{A}_{143}+\frac{1}{V_{1235}} \vec{A}_{235} \cdot \vec{A}_{132}\right] \\
A_{55}= & \frac{D}{36}\left[\frac{1}{V_{1245}}\left\|\vec{A}_{142}\right\|^{2}+\frac{1}{V_{2345}}\left\|\vec{A}_{243}\right\|^{2}+\frac{1}{V_{1345}}\left\|\vec{A}_{143}\right\|^{2}\right. \\
& \left.+\frac{1}{V_{1235}}\left\|\vec{A}_{132}\right\|^{2}\right]
\end{aligned}
$$

In general all points couple to all other points for the pyramid. There are conditions under which either $A_{12}$ or $A_{13}$ vanish. These matrix elements would both be zero only under unusual conditions, and then only for a few isolated pyramids.

\section{Ignored and Surface Terms in the $\Gamma$ Functional}

In this section we will consider the terms that were dropped in going from Equation 1 to Equation 2. In addition, we will consider the effects of boundaries on the system. Up until this point we have been treating the system as infinite in extent. Treating the system as finite will introduce a surface integral term into Equation 1.

In order to discover the surface terms we must re-derive Equation 1 from the original diffusion equation,

$$
-\vec{\nabla} \cdot D \vec{\nabla} \phi+\sigma_{a} \phi-Q=0,
$$

using the weighted residual method.

In this method we construct a functional, $W$, and find a solution to the differential equation, $\mathcal{L}(\phi)=0$ by solving

$$
\begin{aligned}
W & =\int \delta \phi \mathcal{L}(\phi) d^{3} x \\
& =0 \forall \delta \phi .
\end{aligned}
$$

For the diffusion equation this becomes

$$
\begin{aligned}
W & =\int \delta \phi\left(-\vec{\nabla} \cdot D \vec{\nabla} \phi+\sigma_{a} \phi-Q\right) d^{3} x \\
& =\int\left(D \vec{\nabla} \delta \phi \cdot \vec{\nabla} \phi+\delta \phi\left(\sigma_{a} \phi-Q\right)\right) d^{3} x-\int_{S} \delta \phi D \vec{\nabla} \phi \cdot \hat{n} d^{2} x,
\end{aligned}
$$

where we have integrated by parts to obtain the surface integral. Using Marshak boundary conditions,

$$
D \vec{\nabla} \phi \cdot \hat{n}=2 J-\frac{\phi}{2},
$$


the expression for $W$ becomes

$$
W=\int\left(D \vec{\nabla} \delta \phi \cdot \vec{\nabla} \phi+\delta \phi\left(\sigma_{a} \phi-Q\right)\right) d^{3} x-\int_{S} \delta \phi\left(2 J-\frac{\phi}{2}\right) d^{2} x
$$

In order to produce a functional, $\Gamma[\phi]$, that when minimized produces Equation 62 we equate the variation of $\Gamma[\phi]$ to the terms in Equation 62 . The variation of a functional $\Gamma[\phi]$ is

$$
\delta \Gamma=\frac{\partial \Gamma}{\partial \phi} \delta \phi+\sum_{i=1}^{3} \frac{\partial \Gamma}{\partial \frac{\partial \phi}{\partial x_{i}}} \frac{\partial \delta \phi}{\partial x_{i}} .
$$

It is easily shown that the variation of the following functional,

$$
\Gamma[\phi]=\int\left(\frac{1}{2} D \vec{\nabla} \phi \cdot \vec{\nabla} \phi+\frac{1}{2} \sigma_{a} \phi^{2}-Q \phi\right) d^{3} x+\int_{S}\left(\frac{1}{4} \phi^{2}-2 J \phi\right) d^{2} x,
$$

reproduces Equation 62.

With this functional Equation 3 becomes

$$
\begin{aligned}
\Gamma\left(\phi_{1}, \phi_{2}, \ldots\right)= & \sum_{k} w_{k}\left(\frac{1}{2} D\left\|\vec{\nabla}_{k} \phi\right\|^{2}+\frac{1}{2} \sigma_{a} \phi_{k}^{2}-Q_{k} \phi_{k}\right) \\
& +\sum_{l} a_{l}\left(\frac{1}{4} \phi_{l}^{2}-2 J \phi_{l}\right)
\end{aligned}
$$

where the sum over $l$ is over corners on the boundary of the system, and the sum of $a_{l}$ over the corners on the face of a cell equals the area of that face.

The variation of $\Gamma[\phi]$ proceeds as before, by Equation 6 . Using the above equation for $\Gamma$ the volume contribution becomes

$$
\frac{\partial \Gamma}{\partial \phi_{i}}=\sum_{j}\left(A_{i j}+w_{i} \sigma_{a} \delta_{i j}\right) \phi_{j}-w_{i} Q_{i},
$$

where the $A_{i j}$ is the matrix element calculated for the appropriate cell type in the previous sections. The surface contribution is

$$
\frac{\partial \Gamma}{\partial \phi_{i}}=\sum_{j}\left(\frac{a_{i}}{2} \delta_{i j}\right) \phi_{j}-a_{i} 2 J .
$$

There are two cases for the surface term, one when the surface face is a quadralateral, the other when the surface face is a triangle. For the quadralateral case, the equation for $\frac{\partial \Gamma}{\partial \phi_{i}}$ becomes

$$
\frac{\partial \Gamma}{\partial \phi_{i}}=\sum_{j}\left(\frac{1}{8}\left\|\vec{A}_{i}\right\|^{2} \delta_{i j}\right) \phi_{j}-\frac{1}{2}\left\|\vec{A}_{i}\right\|^{2} J
$$




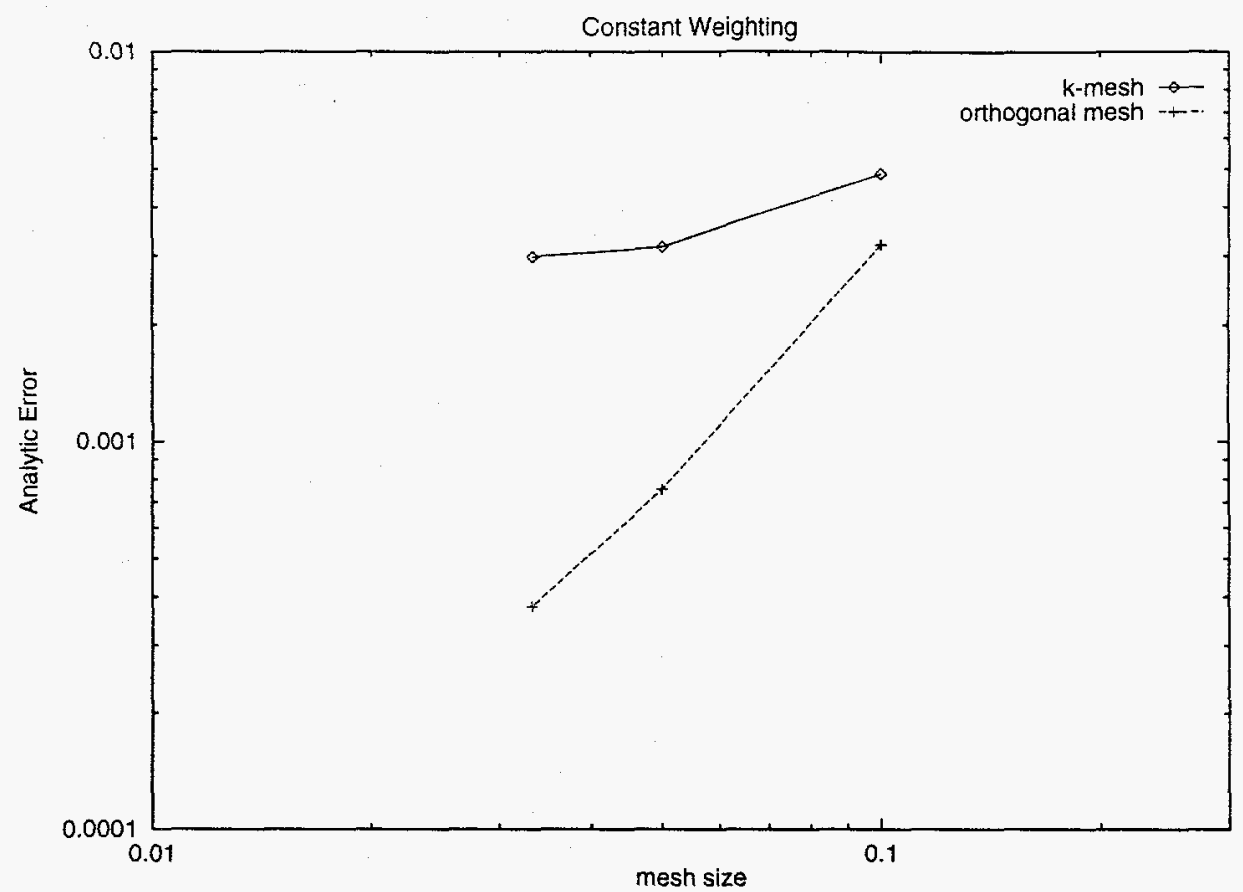

Figure 5: Analytic Error for K-mesh $=.2$ and orthogonal mesh for $-D \frac{\partial^{2} \phi}{\partial x^{2}}-$ $Q x^{2}=0$ with $D=\frac{1}{30}, Q=1$, and $J_{0}=J_{1}=0, w_{k}$ constant weighted.

where $\vec{A}_{i}$ is the area vector created from the two edge vectors at corner $i$ on the face. For the triangal case, the equation for $\frac{\partial \Gamma}{\partial \phi_{i}}$ becomes

$$
\frac{\partial \Gamma}{\partial \phi_{i}}=\sum_{j}\left(\frac{1}{6}\left\|\vec{A}_{i}\right\|^{2} \delta_{i j}\right) \phi_{j}-\frac{2}{3}\left\|\vec{A}_{i}\right\|^{2} J,
$$

where $\vec{A}_{i}$ is the area vector for the triangle.

The volume contribution, Equation 66, is composed for each cell in the system. The surface contribution, either Equation 68 or Equation 69, is composed for each face on the boundary of the system. These equations can be seen to take the form of a matrix equation,

$$
\mathbf{A x}=\mathbf{b},
$$

with $\mathbf{x}$ containing the vector of $\phi_{i} \mathbf{s}$, and the right hand side, $\mathbf{b}$, containing the $Q_{i}$ and $J$ terms. 


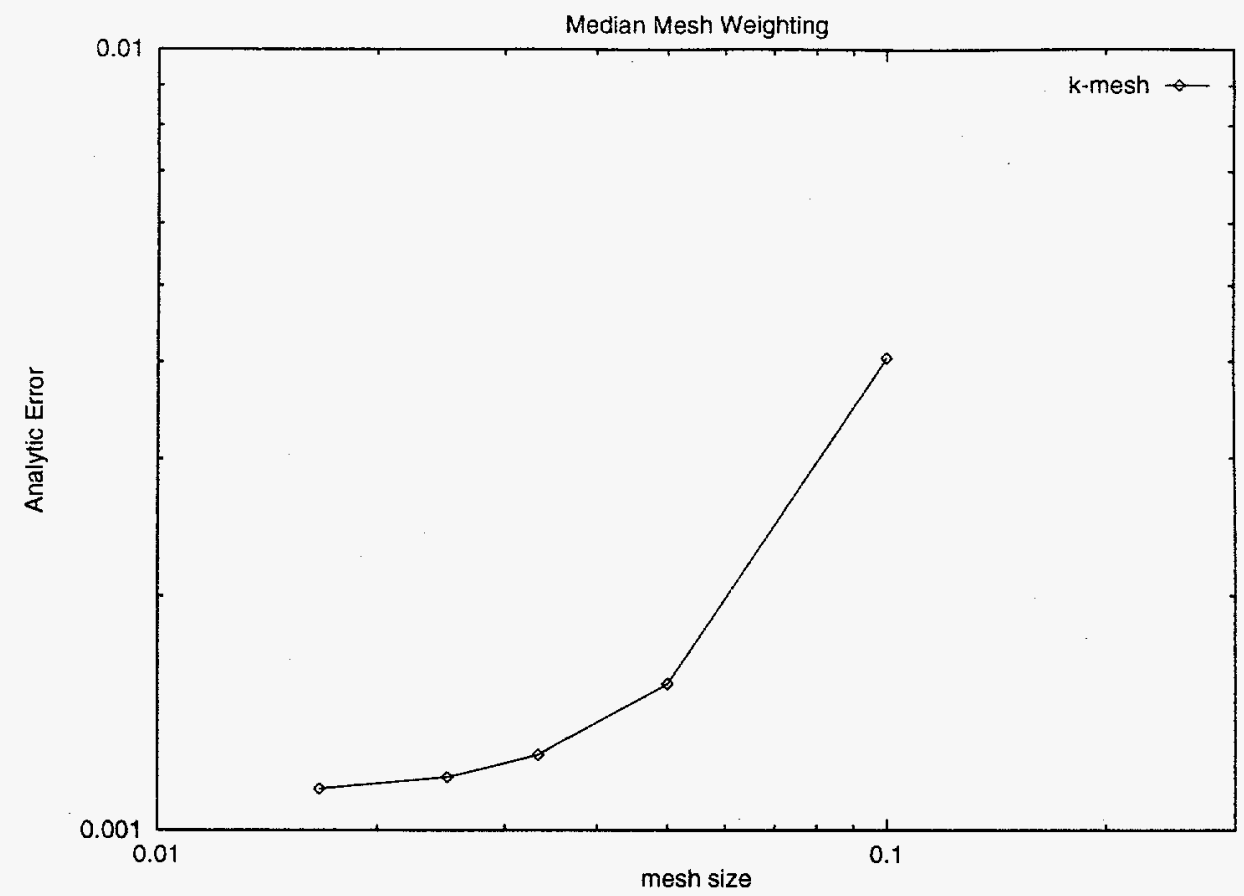

Figure 6: Analytic Error for K-mesh $=.2$ for $-D \frac{\partial^{2} \phi}{\partial x^{2}}-Q x^{2}=0$ with $D=\frac{1}{30}$, $Q=1$, and $J_{0}=J_{1}=0, w_{k}$ median mesh weighted.

\section{Results}

In this section we will present results for hexahedral meshes, and three weighting methods. The analytic error, defined as

$$
\text { Analytic Error }=\left[\frac{\sum_{i}\left(\phi_{i}-\phi\left(\vec{x}_{i}\right)\right)^{2}}{\sum_{i} \phi\left(\vec{x}_{i}\right)^{2}}\right]^{\frac{1}{2}},
$$

is plotted in Figures 5, 6, and 7 .

In Figure 5 the weights, $w_{k}$, are constant. Results are shown for a random mesh, and an orthogonal mesh. The orthogonal mesh shows second order convergence. The convergence is less than second order for the random mesh.

In Figure 6 the weights, $w_{k}$, are the median mesh volumes surrounding the $k$ th point. Results are shown for a random mesh. The convergence is again less than second order for the random mesh.

In Figure 7 the weights, $w_{k}$, are the parallelpiped volumes surrounding the $k$ th point, c.f. Equation 44 . Results are shown for a random mesh. The convergence, in this case, is second order for the random mesh. We have chosen parallelpiped volume weights for this reason.

The linear solution is not recovered using these weights. Using a random mesh, the $20 \times 20 \times 20$ linear problem obtained an analytic error of $7.10 \times 10^{-4}$. In 


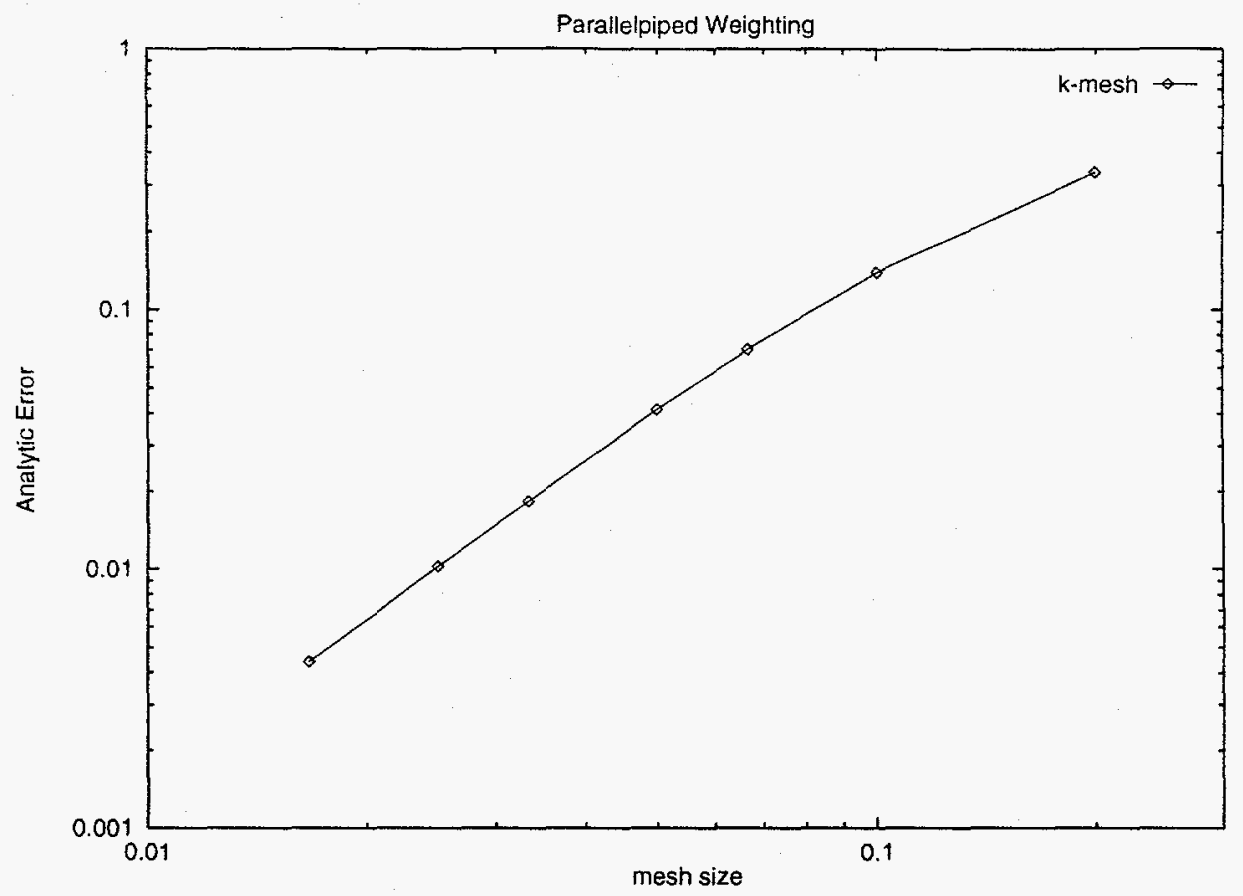

Figure 7: Analytic Error for K-mesh $=.2$ for $-D \frac{\partial^{2} \phi}{\partial x^{2}}+\sigma_{a} \phi-Q x^{2}=0$ with $D=\frac{1}{30}, \sigma_{a}=10, Q=1, J_{0}=0.5$, and $J_{1}=1.0, w_{k}$ parallelpiped weighted.

order to demonstrate that this error was not due to roundoff, we ran the same problem with

$$
w_{i}=\frac{1}{8} V_{i}
$$

It can be shown that this choice of weighting will produce the exact linear solution, though it will yield incorrect results for the general diffusion equation. With this weighting we obtained an analytic error of $1.44 \times 10^{-14}$. This points out the inability to determine the exact linear equation with the preferred weights from Equation 44 even though we obtain second order convergence. 